\title{
Successfully Treated Multifocal Chondrosarcoma in an Adult - A Case Report
}

Arun Vasudevan ${ }^{1 *}$, Sreejith G Nair ${ }^{1}$, Steffi Chacko ${ }^{1}$, Saikumar Soman ${ }^{1}$, Sindhu Nair P ${ }^{2}$, Rani Rajasekharan ${ }^{2}$, Bhaskar Subin Sugath ${ }^{3}$, Manu Paul ${ }^{3}$

${ }^{1}$ Department of Medical Oncology, Regional Cancer Centre Thiruvananthapuram, India

${ }^{2}$ Pathology, Regional Cancer Centre Thiruvananthapuram, India

${ }^{3}$ Surgical Oncology, Regional Cancer Centre Thiruvananthapuram, India

DOI: $10.36347 /$ sjmcr.2020.v08i10.006

| Received: 28.09.2020 | Accepted: 07.10.2020 | Published: 11.10.2020

*Corresponding author: Arun Vasudevan

Abstract

Case Report

Chondrosarcomas are malignant cartilaginous neoplasms with a variable clinical behavior. It often involves the axial skeleton and affects the elderly. It can be primary or secondary chondrosarcoma. Lung is the most common site of metastasis. Here we report a case of multifocal chondrosarcoma involving four different skeletal sites without lung metastasis. Multifocal chondrosarcoma in the absence of lung metastasis is an extremely rare presentation. It is a radioresistant and chemo resistant tumor except for few histologic variants. Surgery is the cornerstone in its management. Complete surgical excision with negative margins of all the involved sites is the preferred treatment modality. In this case it was possible to completely resect all the sites of disease involvement.

Keywords: Chondrosarcoma, multifocal, scapula, humerus, coracoid, acromion.

Copyright (C) 2020 The Author(s): This is an open-access article distributed under the terms of the Creative Commons Attribution 4.0 International License (CC BY-NC 4.0) which permits unrestricted use, distribution, and reproduction in any medium for non-commercial use provided the original author and source are credited.

\section{INTRODUCTION}

Chondrosarcomas are malignant cartilaginous neoplasms with diverse clinical picture and accounts for $20 \%$ of primary bone malignancies [1]. It commonly involves the axial skeleton (pelvis, shoulder, and ribs). Multifocal chondrosarcoma is an extremely rare presentation [2]. It can be either primary or secondary. Primary chondrosarcoma arises denovo while secondary arises from a preexisting cartilaginous lesion. Here we report the case of a 36-year-old lady diagnosed with multifocal chondrosarcoma involving four skeletal sites who was successfully treated with complete surgical resection of all the involved sites.

\section{Case Report}

A 36 old lady was evaluated for left shoulder pain of 3 months duration. Clinical examination was normal except for tenderness involving upper end of humerus without any restriction of movement. Plain Xray of left shoulder joint was normal. Magnetic Resonance Imaging (MRI) of left shoulder showed altered marrow signals with mild to moderate contrast enhancement involving the proximal metadiaphysis of humerus, coracoid process and acromion of the scapula, appearing hypointense on T1 weighted images and T2 weighted fat saturated images. There was no bone expansion, cortical break, soft tissue component or gleno humeral joint involvement (Figure 1 and 2). 


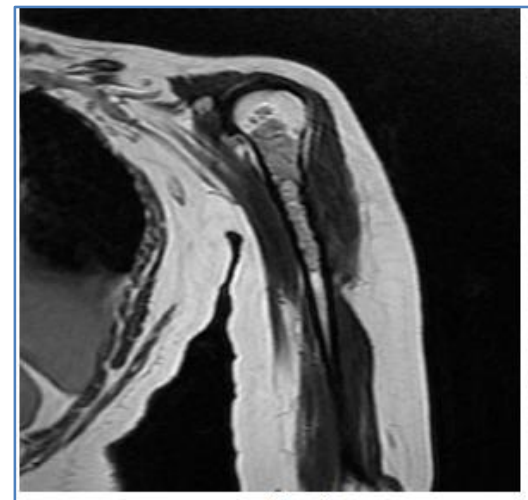

Fig-1

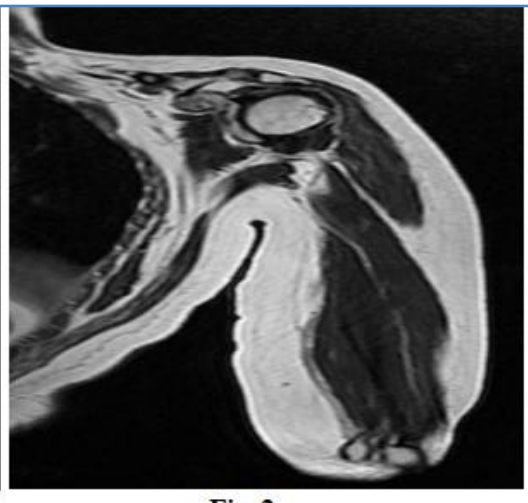

Fig-2

Fig-1 and 2: MRI Left shoulder - Coronal T2 images showing altered marrow intensity lesions involving the humerus and coracoid process

Computerized Tomography scan of Thorax and abdomen showed expansile bony lesions involving the acromion, coracoid process of left scapula, lower end of sternum and long segment endosteal scalloping of left humeral meta-diaphysis with chondroid type calcification, suggestive of synchronous tumors. There was no evidence of lung metastasis. Bone scan also showed tracer uptake involving above mentioned areas. Image guided percutaneous biopsy was done from all the four sites which was reported as Conventional chondrosarcoma grade 2 (Figure 3 and 4). She was further taken up for definitive treatment. She underwent wide excision of all the four lesions. Intra operatively, tumor involved the proximal humerus, lower sternum, acromion and scapula. Wide excision with preservation of the neurovascular bundle was done and the defect was reconstructed using an endoprosthesis. Post-operative period was uneventful. Final histopathologic report was chondrosarcoma grade 2 involving the humerus, sternum, acromion and coracoid process with negative margins of resection. She was kept on further follow up. She is at present doing well with no evidence of disease at 2 years.

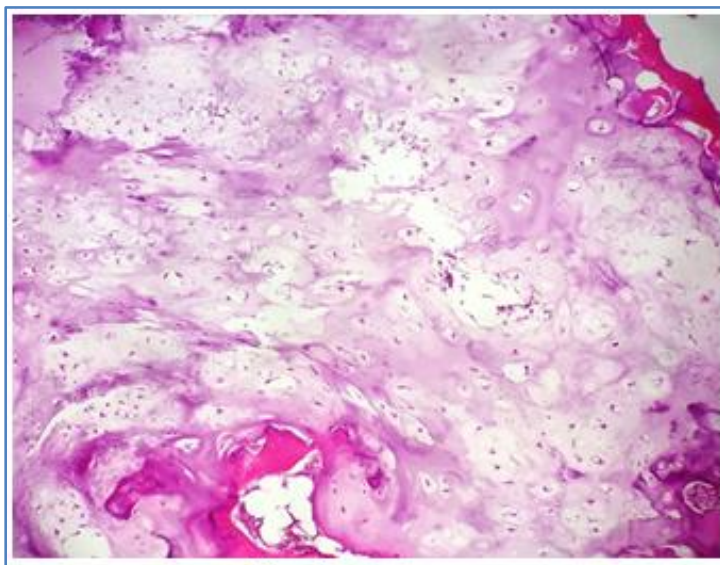

Fig-3

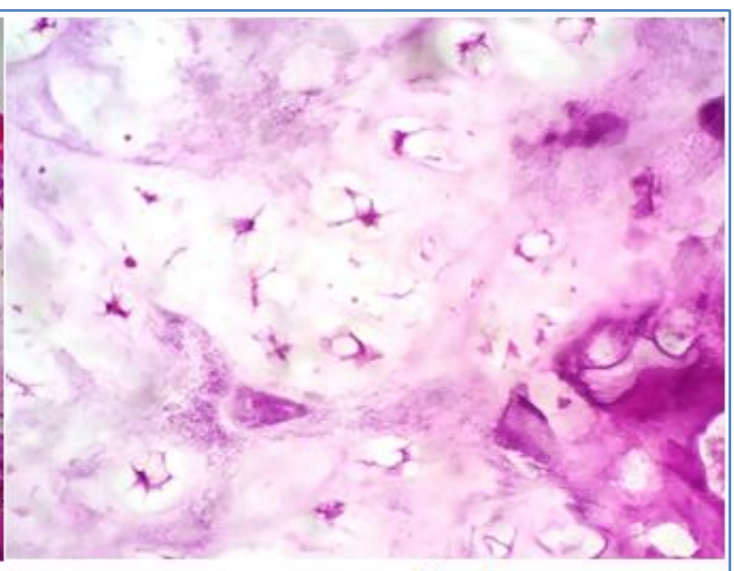

Fig-4

Fig-3: Lobules of hyaline cartilage with increased cellularity (H\&E stain, x100) Figure 4: Atypical chondrocytes in lacunae, with degenerative changes $(H \& E$ stain, $x 400)$

\section{DiscUSSION}

Chondrosarcoma (CS) refers to slow growing primary bone tumors characterized by neoplastic hyaline cartilaginous tissue. These tumors affect patients in the fourth and fifth decade of life with slight male predisposition [3]. Pain is the most common presenting complaint. The duration of symptoms can be very long often. CS is broadly classified into two primary and secondary. Primary chondrosarcoma arises without a benign precursor, while secondary arises from a pre-existing benign tumor (enchondroma or osteochondroma). Primary is again classified into central and peripheral types. Central type arises within the medullary cavity and peripheral from the bone surface. Primary central types include conventional, de- differentiated, clear cell and mesenchymal types. Conventional type is the commonest accounting for 85$90 \%$ of the cases. Most conventional chondrosarcomas are low to intermediate grade tumors with indolent clinical behavior and are of low meta-static potential [4]. The precipitating factors for the development of this tumor are are multiple hereditary exostosis, Ollier's disease, Maffucci's syndrome, Paget's disease of bone, chondromyxoid fibroma, previous irradiation etc.

The plain X-ray can usually provide a clue to the probable histology of a potentially malignant bone lesion [5]. Magnetic Resonance Imaging (MRI) is the preferred local imaging modality in which the lesion appears as high signal intensity lesions on T2-weighted 
images and is preferred as it assesses the size, location, proximity to neurovascular bundle and extent of marrow and soft tissue involvement. Percutaneous biopsy has to be taken from the most aggressive appearing areas, by the surgeon who performs the final surgery. Lung is the commonest site of metastasis. Other sites include regional nodes, bone and liver. It is very unusual to have metastasis in less common sites without lung metastasis. Large tumor and high grade at presentation are risk factors for developing metastasis

Management of chondrosarcoma is based on the type, the grade and the location of the tumor. Patients with grade 1-3 and clear cell variant are treated with primary surgery, while dedifferentiated type and mesenchymal type is treated like high grade bone sarcomas. Surgery is the only curative form of treatment in conventional chondrosarcoma and aims at maximal safe resection with minimal functional disability without compromising the outcome. The options include (1) extensive intralesional curettage (2) wide excision with negative margins or (3) amputation depending on the site and grade of tumor [6]. Intra lesional excision is preferred in grade 1 tumors involving long bones without joint and soft tissue involvement. Wide excision with or without reconstruction is preferred for grade 2 and 3 tumors [7]. Negative margin of resection is one of the most important prognostic factors determining the long-term survival [7]. Being a radio resistant tumor, radiotherapy is considered as primary modality of treatment only if unresectable. Radiotherapy is also recommended after incomplete resection of recurrent tumor and also for palliation of symptoms due to local recurrence [8]. Conventional chondrosarcoma is not chemotherapy sensitive but chemotherapy has role in dedifferentiated and mesenchymal forms [9].

Metastatic disease at presentation is treated with surgical resection of all lesions and radiotherapy to unresectable sites. Our patient presented with disease involving four different skeletal sites. A successful resection of all the lesions with negative margins followed by reconstruction was done. Patients with widespread metastatic disease may be either enrolled in clinical trial or can be offered palliative chemotherapy with agents like Doxorubicin and Cisplatin. Chemotherapy refractory patients may be given tyrosine kinase inhibitors like Dasatinib [10].

\section{CONCLUSION}

De-novo Multifocal Chondrosarcoma is a rare presentation. Multifocal chondrosarcoma in the absence of lung metastasis is still rare. It often affects patients in the fourth and fifth decade of life. Surgery is the cornerstone in its management. Grade of the tumor determines the type of primary surgery. Surgical resection of all the lesions with negative margins is the treatment of choice and must be attempted whenever possible.

\section{REFERENCES}

1. Thorkildsen J, Taksdal I, Bjerkehagen B, Haugland HK, Borge Johannesen $\mathrm{T}$ and Viset $\mathrm{T}$. Chondrosarcoma in Norway 1990-2013; an epidemiological and prognostic observational study of a complete national cohort. Acta Oncol. 2019 Mar;58(3):273-282

2. Kavitha P, Rajanikanth BR, Vineeth K, Chandrashekar R, Preeti S. An unusual case report of multifocal chondrosarcoma of the mandible. Journal of Oral and Maxillofacial Surgery, Medicine, and Pathology. 2018 March; 30(2):129133

3. Van Praag Veroniek VM, Rueten-Budde AJ, Ho V, Dijkstra PDS, Study group Bone and Soft tissue tumours (WeBot);Fiocco M, van de Sande MAJ. Incidence, outcomes and prognostic factors during 25 years of treatment of chondrosarcomas. Surg Oncol. 2018 Sep;27(3):402-408.

4. Heck RK, Jr, Peabody TD, Simon MA. Staging of primary malignancies of bone. CA Cancer J Clin. 2006;56(6):366-75.

5. Ollivier L, Vanel D, Leclère $\mathrm{J}$. Imaging of chondrosarcomas. Cancer Imaging. 2003 Oct 22;4(1):36-8

6. Barnes R, Catto M. Chondrosarcoma of bone. J Bone Joint Surg [Am] 1966; 48:729-64.

7. Fiorenza F, Abudu A, Grimer RJ, Carter SR, Tillman RM and Ayoub K. Risk factors for survival and local control in chondrosarcoma of bone. J Bone Joint Surg Br. 2002;84(1):93-9

8. Hans Gelderblom, Pancras CW Hogendoorn, Sander D Dijkstra, Carla S van Rijswijk, Augustinus D Krol, Antonie HM Taminiau, Judith V M G Bovée. The Clinical Approach towards Chondrosarcoma. 2008 Mar;13(3):320-9

9. Cesari M, Bertoni F, Bacchini P, Mercuri M, Palmerini E, Ferrari S. Mesenchymal chondrosarcoma. An analysis of patients treated at a single institution. Tumori. 2007 SepOct;93(5):423-7

10. Scott M Schuetze, Vanessa Bolejack, Edwin Choy, Kristen N Ganjoo, Arthur P Staddon. Phase 2 Study of Dasatinib in Patients with Alveolar Soft Part Sarcoma, Chondrosarcoma, Chordoma, Epithelioid Sarcoma, or Solitary Fibrous Tumor. 2017 Jan 1;123(1):90-97. 